\title{
Sulla « rugosità » del paesaggio
}

\section{Roberta Cevasco}

\section{(2) OpenEdition}

\section{Journals}

\section{Edizione digitale}

URL: http://journals.openedition.org/edl/517

DOI: $10.4000 /$ edl.517

ISSN: 2296-5084

\section{Editore}

Université de Lausanne

\section{Edizione cartacea}

Data di pubblicazione: 15 mai 2013

Paginazione: 323-344

ISBN: 978-2-940331-32-1

ISSN: 0014-2026

\section{Notizia bibliografica digitale}

Roberta Cevasco, «Sulla « rugosità » del paesaggio », Études de lettres [Online], 1-2 | 2013, online dal 15 mai 2016, consultato il 18 décembre 2020. URL : http://journals.openedition.org/edl/517 ; DOI : https://doi.org/10.4000/edl.517 


\section{SULLA «RUGOSITÀ» DEL PAESAGGIO ${ }^{1}$}

La rugosité du paysage géographique est une métaphore de sa matérialité. Contrairement à l'approche dominante qui pose au centre la perception, la prise en charge de la rugosité permettra d'identifier de nouvelles sources et méthodes pour l'étude historique des paysages ruraux par les traces matérielles. Le centre de la discussion est la contribution de l'écologie historique selon sa formulation dans le contexte italien des années 1990, lorsque on a établi un dialogue avec la microhistoire (dans sa version finale: nouvelle histoire locale). Quelques exemples de microanalyse géographique et historique de paysages ruraux individuels ont été pris à partir du récent Catalogo Nazionale dei Paesaggi Rurali Storici, par des cas d'étude soulignant les processus historiques de biodiversification.

\section{Il geografo sul terreno: il punto di vista dell'ecologia storica}

Le rughe sono un segno del tempo. La rugosità non è una caratteristica delle immagini. L'approccio storico allo studio del paesaggio geografico da qualche tempo ha imboccato un percorso che torna a porre la materialità del paesaggio e il suo spessore storico - le sue rughe - al centro delle analisi in luogo del prevalente interesse percettivo proprio della geografia culturale post-moderna, alimentato dalle sole tematiche dell'immagine e della rappresentazione. L'approccio "culturalista»,

I. Questo testo riprende l'intervento al Seminario-Laboratorio internazionale sul paesaggio, San Biagio della Cima, 15 gennaio 2011. La metafora della rugosità del paesaggio mi è stata suggerita dal convegno geografico «Di monti e di acque. Le rughe e i flussi della Terra. Paesaggi, cartografie e modi del discorso geostorico ", Università degli Studi di Trento, CISGE, dicembre 2010. 
infatti, tende non solo a rinchiudere la geografia umana entro l'orizzonte delle rappresentazioni simboliche ma esclude le possibilità di decifrazione realistica del paesaggio culturale in quanto oggetto geografico ${ }^{2}$. E evidente il rischio che $\mathrm{i}$ "paesaggi culturali» siano identificati e valorizzati solo per la dimensione simbolica e percettiva banalizzando i processi storico-ambientali che pure li hanno costruiti - e li continuano a costruire - nella loro materialità e complessità. Il rischio è presente anche nel testo della Convenzione Europea del Paesaggio che fonda il concetto "politico-amministrativo" di paesaggio su definizioni di tipo percettivista e non dedica, non riconosce, uno spazio specifico alla vasta e ricca problematica dei paesaggi rurali o agrari ${ }^{3}$.

Questa «smaterializzazione del paesaggio come oggetto della ricerca geografica» è stata sottolineata con chiarezza anche in occasione del recente dibattito su paesaggio e musei apparso sulla Rivista Geografica Italiana $^{4}$. I geografi, sottolinea Maria Luisa Sturani, sono sempre meno visibili e interessati a fornire apporti analitici nello studio delle dinamiche territoriali: una invisibilità che si manifesta purtroppo nel momento in cui, nel più vasto ambito europeo, la problematica dei paesaggi culturali (ed anche rurali ed agrari) si iscrive nella prospettiva del patrimonio rurale 5 .

Sintomatica della frattura consumatasi attorno al tema del paesaggio tra orientamenti geo-storici e culturalisti, e a sua volta potenzialmente foriera del ridursi degli spazi di collaborazione con i musei, è la vera e propria rimozione del paesaggio materiale dall'ambito della riflessione sull' heritage, che coinvolge la disciplina negli anni '90. [...] Nelle enunciazioni più estreme di tale visione non si dà paesaggio se non come rappresentazione e la geografia si auto-esclude dall'analisi del patrimonio culturale quale documento storico: una rinuncia che [...] ha esiti sconcertanti là dove essa viene tacitamente estesa anche al paesaggio

2. M. Quaini, L'ombra del paesaggio, p. 91.

3. D. Moreno, C. Montanari, "Il lato "oscuro" del paesaggio».

4. Si tratta di un numero monografico della Rivista Geografica italiana curato da Maria Luisa Sturani (CXVI, fasc. 4, dicembre 2009).

5. Per una proposta matura, ma totalmente debitrice all'etnografia rurale francese, cfr. la guida Guide Européen d'observation du patrimoine rural prodotta dal CEMAT (2003) e tradotta in italiano a cura di Maria Chiara Zerbi (2007); URL: http://www. coe.int/t/dg4/cultureheritage/heritage/cemat/versionguide/Francais.pdf, consultato il 10.02.2012. 
come patrimonio di cultura materiale, destituendo di interesse e legittimità una lunga e fruttifera stagione di ricerche geografiche e le sue potenzialità applicative. [...] In fondo, trattare il paesaggio come idea è la sola condizione per poterlo "chiudere» in un museo $^{6}$.

Perché non considerare le rughe del paesaggio culturale, non riprendere cioè nel discorso del geografo anche il lato materiale e concreto di quelle «impronte» o «eredità culturali» alla sua base? Perché avallare i limiti metodologici dell'approccio semiologico/culturalista rispetto alle possibilità di analisi storico-geografica degli spazi concreti? Il geografo, nell'evitare queste domande, riconsegna la pianificazione e la gestione degli spazi su cui insiste il patrimonio ambientale e culturale dei paesaggi rurali alla filosofia della conservazione naturalistica. Oggi sono possibili nuove risposte perché sono disponibili nuovi strumenti per il lavoro geografico di terreno.

Lapproccio storico-analitico al paesaggio, per ora, è poco più di un sentiero ma la via è aperta da anni. Le prime mosse sono derivate dall'historical ecology. Sono stati proprio i naturalisti inglesi, che non hanno mai abbandonato i field studies nella loro pratica di lavoro, a riconoscere le risorse ambientali non come il semplice risultato di dinamiche naturali, evolutive, ma come il prodotto di un processo storico e sociale. Se la copertura vegetale è un $\operatorname{artifact}^{7} \mathrm{e}$ " un bosco è parte della società locale" ${ }^{8}$, ecosistemi e sistemi sociali non possono essere studiati separatamente. Proprio dall'analisi della struttura materiale, biologica, ecologica e storica ad esempio di uno specifico individual wood emerge il fascio di relazioni che lega un gruppo sociale ad un paesaggio individuale. Nella prospettiva dell'ecologia storica questo fascio si infittisce e si scoprono nuovi spazi per la geografia interessata alla dimensione sociale dei fenomeni che studia. Da qui, in Europa, l'interesse per il potenziale, e nodale, nuovo ruolo della geografia nell'analisi del patrimonio ambientale e rurale e nelle politiche della sua gestione, che qualche anno fa George Bertrand ha chiamato le retour du géographique.

Nell'Europa mediterranea, tuttavia, si fa ancora fatica a riconoscere la dimensione processuale, storica, dei paesaggi rurali. L'idea del bosco come manufatto e della biodiversità come costruzione storica, cui si farà

6. M. L. Sturani, «Paesaggio e musei», p. 390-392.

7. O. Rackham, Trees and Woodland in the British Landscape.

8. G. F. Peterken, «Historical Approach to Woodland Ecology and Management». 
cenno nella seconda parte, sono ancora lontani: i naturalisti italiani, per lo più, continuano ad essere interessati agli «assetti originari» della vegetazione e ai "paesaggi primordiali " ${ }^{9}$ come riferimento per le analisi del presente. Queste posizioni persistono nei metodi di una «ecologia del paesaggio " priva di analisi storica, con i suoi presupposti di «naturalità " e la conseguente distinzione tra paesaggi senza «influenza umana" (versione moderna del pristine environment) e con «influenza umana» (in genere assunti come degradati o semplificati). Così posta, la questione è ancora nei termini (cfr. "influenza») in cui era discussa dai geografi negli anni '20, come ad esempio nella nota critica di Lucien Febvre ${ }^{10}$ alle tesi deterministiche di Miss Helen Churchill Semple.

L'«approccio storico all'ecologia dei siti», come si vedrà, è lentamente maturato nella geografia italiana ma, in effetti, non ha ancora trovato una sistemazione teorica. A ben vedere, lo stesso si può dire per i lavori suscitati in Gran Bretagna e Norvegia da botanici come Oliver Rackham, George Peterken, Keith Kirby soprattutto sul tema degli ancient woodlands o da geografi come John Sheail e Charles Watkins e storici come Ross Balzaretti ${ }^{11}$. Ben più definito appare lo statuto dell' historical ecology negli Stati Uniti dove possiamo individuare, tra gli approcci di maggior interesse, quelli di antropologi/etnobotanici come William Balée, di esperti in ecologia storica e gestione del fuoco come Stephen Pyne, di botanici con forte propensione per la dimensione storica come Emily Russell ${ }^{12}$. Il

9. Questa ecologia dei paesaggi primordiali pare lontana sia dalle posizioni dell'ecologia storica sia dalle più recenti acquisizioni dell'ecologia strutturale, per cui si vedano, ad esempio in una rivista come Nature, le critiche contro quegli ecologi che trattano il "passato" (o meglio una "situazione primigenia») come un referente teorico a cui si dovrebbe tornare (cfr. la recensione di Jon Christensen al volume di Steve Nicholls, Paradise Found: Nature in America at the Time of Discovery, University of Chicago Press, 2009, in Nature 459 [14 maggio 2009], p. 167 sq.). Si segnala la posizione di Nature in quanto maturata nell'ambito delle scienze ambientali «dure». Per le posizioni assunte dai geografi nei confronti della "naturalità/naturalizzazione», a cui in generale la disciplina è prona nei momenti della pianificazione, è necessario distinguere caso per caso. Si può già anticipare che sono molto pochi i geografi che hanno percorso l'approccio storico al problema. Per una riflessione sul "disarmante» naturalismo contemporaneo si veda G. Marrone, Addio alla Natura. Una proposta di dibattito su bioetica e geografia si ritrova in Da Pozzo, «Bioetica e geografia».

Io. L. Febvre, La terre et l'évolution humaine.

II. R. Balzaretti, «Victorian Travellers, Apennine Landscapes and the Development of Cultural Heritage in Eastern Liguria, c. 1875-1914».

I2. E. W. B. Russell, People and the Land through Time. 
recente Convegno dell'International Union of Forest Research Organizations (IUFRO) dal titolo Frontiers in Historical Ecology ${ }^{13}$, ha presentato un quadro delle ricerche europee e statunitensi in ecologia storica selezionando i lavori, sembra, con maggiore propensione per la modellizzazione quantitativa ${ }^{14}$. Complessivamente l'ecologia storica che prevale a Birmensdorf è quella di matrice nord-americana in larga espansione anche in Europa. Oggi, quando in geografia si fa riferimento all' historical ecology si rischia l'adesione a questa recente tendenza confondendola con l'esperienza inglese.

Esiste invece, nella storia dei rapporti con le scienze geografiche, anche una via italiana all'ecologia storica, che potremmo propriamente definire "geografica» con riferimento alle posizioni di partenza di un Lucio Gambi. Le condizioni per un «approccio storico microanalitico » ai problemi geografici, infatti, si prefigurano nell'incontro tra una geografia umana storica che si richiama a Lucio Gambi e a Massimo Quaini e l'ecologia storica di derivazione inglese. Cemento della problematica - se questo ruolo si può domandare ad un paradigma storiografico debole per scelta - è stata negli anni '90 la "storia locale» di Edoardo Grendi, che come si accennerà, ha una diretta discendenza, al pari dell' historical eco$\operatorname{logy}$, da una comune topographical history. Una prospettiva che consente di superare il dualismo tra problematica ecologica e storica ${ }^{15}$, anche attraverso una nuova produzione e lettura delle «fonti di terreno ${ }^{16}$.

13. Organizzato dal Swiss Federal Institute for Forest, Snow and Landscape Research (WSL) a Birmensdorf (Svizzera) il 30 agosto - 2 settembre 2011 (URL: http:// www.wsl.ch/dienstleistungen/veranstaltungen/veranstaltungskalender/frontiers-2011/ Program.pdf, consultato il 10.02.2012).

I4. Si veda ad esempio il titolo della seconda sessione, presieduta dal belga Martin Hermy, «Beyond case studies in historical ecology: the search for general patterns of ecosystem changes». La folta presenza di ricercatori svedesi è da connettere all'effetto "Carole Crumley", antropologa all'Università della North Carolina e pioniera nella pratica dell' historical ecology e della landscape archaeology statunitense, attualmente guest researcher allo Stockholm Resilience Centre.

15. Che la netta separazione gambiana tra Geografia ecologica e Geografia umana vada ridiscussa è oggi ampiamente accettato tra i geografi, si veda in proposito anche l'intervento di Giuseppe Dematteis nel numero di Quaderni Storici curato da Massimo Quaini e dedicato allo stesso Gambi con il titolo «Nuovi percorsi della geografia umana in una storia non lineare».

I6. D. Moreno, Dal documento al terreno; R. Cevasco, Memoria verde; D. Moreno, C. Montanari, «Il lato "oscuro" del paesaggio»; M. Quaini, I paesaggi italiani. 
Si tratta di un'esperienza che si è sviluppata parallelamente in ambito archeologico e naturalistico già negli anni ' 70 quando questi diversi approcci allo studio dell'ambiente hanno trovato una loro unità. Come ricorda Edoardo Grendi in una monografia destinata a sistematizzare la storiografia locale in Liguria, già con le proposte di Tiziano Mannoni, un naturalista prestato per lunghi decenni all'archeologia medievale e post-medievale, il suolo stesso diventa - per l'archeologo (e per lo storico della località) - piena fonte storica, e quindi anche la vegetazione:

[...] nell'ambito degli studi sul terreno, non vi è dubbio che il metodo archeologico-naturalistico di Tiziano Mannoni abbia un forte significato di rottura epistemologica nel ricondurre al rango di fonte storica il terreno stesso e, su questa scia, anche la vegetazione, entrando in rotta di collisione coi naturalisti ${ }^{17}$.

Va detto che in Italia, pur proposto almeno dagli ultimi decenni del secolo scorso ${ }^{18}$ l'approccio storico microanalitico, fortemente multidisciplinare, non ha trovato grande seguito tra naturalisti, geografi e storici. Forse perché, oltre a richiedere tempi di analisi molto lunghi, l'ecologia storica - come hanno sottolineato di recente William Balée e Clark Erickson ${ }^{19}$ - non è un paradigma nel senso di Thomas Kuhn ${ }^{20}$, né una disciplina, ma piuttosto un programma di ricerca, con alla base un'idea radicalmente nuova: le società umane sin dalla Preistoria hanno per lo più aumentato la ricchezza della "natura» promuovendo la biodiversità, la fertilità dei suoli (cambiando il chimismo e la tessitura), l'eterogeneità delle forme della terra. Certamente vi sono processi che hanno anche diminuito la biodiversità (in particolari momenti storici, e certamente negli ultimi secoli in molte aree della Terra), ma quest'ultima osservazione, che riguarda la società industriale occidentale, ricordano i nostri autori, non costituisce una novità ${ }^{21}$.

17. E. Grendi, Storia di una storia locale, p. 177.

I8. D. Moreno, Dal documento al terreno.

19. W. Balée, C. Erickson, "Time, Complexity and Historical Ecology».

20. Th. Kuhn, The Structure of Scientific Revolutions.

2I. W. Balée, C. Erickson, "Time, Complexity and Historical Ecology» (URL: http://repository.upenn.edu/anthro_papers/15/, consultato il 15.02.2012). William Balée è un antropologo statunitense alla Tulane University (New Orleans) che ha maturato un particolare interesse anche teorico verso le basi dell' historical ecology. 
Tornando alla proposta di un "approccio storico microanalitico" ai problemi geografici, occorre sottolineare un particolare collegamento. L' historical ecology in Gran Bretagna si afferma negli anni '70 nell'ambito degli studi per la conservazione ambientale anche sulla scia del forte prestigio scientifico della local history, ai cui metodi e fonti fa riferimento per affrontare la dimensione storica dei problemi che incontra sul terreno. Come sottolinea Edoardo Grendi nel suo programma per una nuova storia locale, la local o topographical history:

si è sviluppata anche come disciplina di prestigio accademico, nell'immediato dopoguerra, fondendo l'approccio topografico tradizionale con quello storico-economico recente e acquistando «momento" proprio per la convergenza delle "discipline del territorio" (archeologia, botanica, ecc.) che da noi sono rimaste invece legate a una prospettiva naturalistica atemporale 22 .

E ancora :

Il risultato raggiunto dalla local history inglese è stato duplice: non solo si è mantenuto e rifondato il rapporto fra uomini e cose, fra storia e scienze del territorio; ma si è consentito il superamento di questo dualismo aprendo la natura alla comune dimensione culturalizzante della temporalità storica. Il paesaggio locale specifico risulta così una fonte storica, un palinsesto leggibile nei termini di una stratigrafia degli interventi umani; e l'esperienza di sito che è l'archeologia può esser chiarita, nella sua successione stratigrafica, come una realtà testimoniale a scala multitemporale senza cesure e scarti; non solo le pietre delle città ma anche la vegetazione, il terreno possono essere uniformemente letti e studiati come manufatti [...]. In questo modo proprio la local history ha stimolato una profonda revisione e rifondazione delle scienze naturali, non più congelate nei vecchi paradigmi classificatori $^{23}$.

Come fa notare un altro esponente dell'esperienza della microanalisi, Osvaldo Raggio, l'ecologia storica si alimenta di un genere storiografico (local o topographical history) che è rimasto estraneo agli studi storici e geografici nel nostro paese fino a quando, in tempi recenti, la rielaborazione teorica di una «nuova storia locale» è emersa come risultato

22. E. Grendi, Storia di una storia locale, p. 173.

23. Ibid., p. 18 sq. 
scientifico del dibattito, mai chiuso nel corso degli anni 1980, sulla microstoria ${ }^{24}$.

In questo quadro sembra possibile recuperare un nuovo dialogo tra scienze ambientali e scienze geografiche e storiche, anche attraverso il riconoscimento del fatto che le interrelazioni ecosistemiche hanno una durata storica e le loro cronologie (processi di continuità/discontinuità) diventano comparabili con il tempo storico politico e sociale. La problematica della storia delle risorse ambientali emerge come possibile area di incrocio proprio grazie anche al contributo dei metodi e delle fonti dell'archeologia ambientale e della «nuova storia locale» ${ }^{25}$. ̇̀ evidente, infatti, che nell'affrontare lo studio del paesaggio geografico nella sua materialità è necessario allargare lo spettro delle fonti, utilizzando archivi documentari e archivi di terreno, per ricostruire, di volta in volta, e attraverso cronologie fini, i diversi contesti locali, sociali e politici che hanno dato forma ai diversi paesaggi individuali.

Oltre al problema delle fonti, tra gli obiettivi propri dell'approccio geografico-storico microanalitico vi è quello di qualificare i processi di "attivazione» delle risorse ambientali, confrontandosi con l'analisi delle loro implicazioni socio-politiche e identificando le forme storiche, localizzate o locali, che hanno assunto (pratiche di attivazione).

Sin dalla Preistoria le società interagiscono con le risorse ambientali oltre che con esplicite pratiche di produzione anche attraverso "pratiche di attivazione", intenzionali e non intenzionali. Secondo l'approccio dell'ecologia storica le pratiche attivano le risorse vegetali e animali in maniera diretta ${ }^{26} \mathrm{e}$ in maniera indiretta con effetti di «attivazione» - anche inconsapevoli - su altre componenti dell'ecosistema che vengono raggiunte da questi scambi di materia ed energia. Ne è un esempio l'effetto di «esternalità positiva» rilevabile nei sistemi locali di produzione foraggera, come si vedrà esemplificato più oltre.

Il concetto di pratiche di attivazione è un concetto maturato nelle ricerche di terreno dell'ecologia storica e su questo di recente si è aperta

24. O. Raggio, "Microhistorical Approaches to the History of Liguria»; A. Ingold, «Ecrire la nature».

25. R. Cevasco, V. Tigrino, "Lo spazio geografico concreto".

26. Producendo, ad esempio, una "selezione storica» alla scala locale delle specie presenti in un popolamento che è diversa dalla ipotetica o teorica "selezione naturale». Cfr. W. Balée, C. Erickson, «Time, Complexity and Historical Ecology», p. 7. 
una riflessione storiografica. Angelo Torre, in un paragrafo dedicato a Localiser les paysages: un exemple ligure, nota che

Au lieu d'employer une notion purement symbolique de paysage, il est nécessaire, en passant par des cas d'étude - peut-être pourrions-nous dire des «sites d'étude»-, d'appréhender l'étude de l'environnement et de ses dynamiques historiques précises à l'aide de la notion d'«activation des ressources végétales». Cette notion permet de ne pas séparer des éléments de l'univers botanique et organique des pratiques sociales et culturelles, avec leurs valeurs juridiques et relationnelles $[\ldots]^{27}$.

Il concetto di "attivazione " così inteso riguarda sia le risorse ambientali sia le risorse politiche: osservando alla scala del «sito", il ricercatore «rimuove strati di terreno, risale dalle tracce alle pratiche e ne cerca le relazioni con saperi, diritti, relazioni sociali ${ }^{28}$.

Oggi l'approccio geografico-storico microanalitico si propone di «svelare, riscoprire, quei rapporti storici tra società e risorse che la dinamica delle forme del paesaggio rurale - ma ancor più la percezione che ne hanno acquisito le società urbane - spesso velano, oscurano ${ }^{29}$. I problemi della storia delle risorse ambientali vengono affrontati - rispetto alle generalizzazioni a cui ci ha abituato la storia ambientale - ad una scala topografica, adottando un'alta risoluzione spaziale («sito»), temporale e storiografica, quest'ultima intesa come attenzione agli aspetti minuti della configurazione sociale (pratiche, azioni, conflitti). La scala «micro" nella lettura topografica del paesaggio può far emergere questa storia geografica delle risorse ambientali, che ha al centro le relazioni tra gli oggetti geografici e le configurazioni sociali, le loro interazioni e reciproche attivazioni. $\mathrm{Ci}$ si torna ad occupare di uno dei temi e delle categorie centrali della geografia umana con gli strumenti (ad es. nuove fonti) e i metodi analitici che la generazione di Lucio Gambi ed Emilio Sereni non poteva avere a disposizione ${ }^{30}$.

27. A. Torre, "Un "tournant spatial” en histoire?», p. 1144.

28. A. Torre, Luoghi, p. 5.

29. D. Moreno, C. Montanari, «Il lato "oscuro" del paesaggio».

30. Il riferimento è al Catalogo della Mostra documentaria Paesaggi agrari. L'irrinunciabile eredità scientifica di Emilio Sereni promossa da Istituto Alcide Cervi Biblioteca, Archivio Emilio Sereni, Società Geografica Italiana (M. Quaini, Paesaggi agrari). Si veda il contributo di Massimo Quaini e di chi scrive. 
Tra i risultati più promettenti dell'adozione della scala "micro" troviamo dunque le nuove fonti di terreno. Queste - come ad esempio i pollini - sono offerte alla lettura del geografo attraverso l'adattamento di uno strumento convenzionale della ricerca in paleoecologia: l'analisi pollinica. Nel caso in cui sia applicata ai suoli - caso molto particolare ed assai problematico - può essere combinata con i risultati dell'archeologia ambientale e con l'analisi dell'ecologia attuale del sito in una prospettiva non più paleoecologica ma di ecologia storica ${ }^{31}$. Un risultato già previsto alle origini dell'ecologia storica europea quando, in un volume che ha contribuito ai fondamenti dell'approccio ecologico storico nell'Europa settentrionale, si sosteneva l'interesse per la ricerca dell'alta risoluzione spaziale e la scelta di eseguire analisi polliniche alla scala di sito ${ }^{32}$, scelta che trovava già allora piena corrispondenza in modelli scandinavi (D. Moe, M. J. Gaillard, B. Berglund, ecc.).

Il percorso che si propone oggi con la microanalisi geografico-storica è quello di prendere in conto le rugosità, anche le più lievi, del paesaggio. Infatti la ricerca geografica "ha bisogno soprattutto di aderire al terreno nelle sue minime asperità, nei suoi più minuti dettagli» ${ }^{33}$. Massimo Quaini vede le possibilità analitiche di questa nuova storia ambientale praticata dai geografi attraverso poche righe colte da una breve favola di Henrich Heine :

Anche la natura ha una sua storia che è ben diversa dalla storia naturale che ci viene insegnata a scuola. Basterebbe nominare professore straordinario presso una delle nostre università una qualsiasi di quelle lucertole grigie che da secoli vivono nelle fessure delle rocce dell'Appennino e così si verrebbero a sapere le cose più straordinarie ${ }^{34}$.

Una storia diversa, propriamente geografica, delle risorse ambientali e dei paesaggi rurali è diventata oggi possibile, seguendo i singolari dettagli

3I. Le ricerche svolte dal 1996 presso l'Università di Genova dal LASA (Laboratorio interdisciplinare di Archeologia e Storia Ambientale, URL: http://www.dismec.unige. it/lasa/) e nell'ambito della Scuola di dottorato in Geografia storica per la valorizzazione del patrimonio storico-ambientale si basano sull'integrazione di competenze geografico-storiche, botaniche e archeologico-ambientali e trovano applicazione in particolare nella problematica della conservazione ambientale e culturale.

32. H. H. Birks et al. The Cultural Landscape, p. 185.

33. M. Quaini, I paesaggi italiani, p. 138.

34. Ibid., p. 137. 
della topografia, decifrando le tracce iscritte nei suoli, nella pioggia pollinica, nelle piante o nelle code delle lucertole, senza limiti temporali, in direzione contraria alla retorica di letture piatte, astratte e generalizzanti.

\section{Paesaggi rurali di interesse storico}

L'interesse per il geografo di tornare con il lavoro di terreno all'osservazione della componente vegetale dei paesaggi culturali - la loro «memoria verde" - è stato messo al centro di una recente lettura analitica di paesaggi appenninici (ma anche di casi europei) sfuggendo alla categorizzazione tipologica. In questa ricerca i popolamenti vegetali individuali - topograficamente delimitati - sono stati considerati nella loro potenzialità di vere e proprie fonti storiche, memoria in tracce di azioni, interazioni e attivazioni (pratiche di produzione e attivazione) a cui sono stati sottoposti nel tempo ${ }^{35}$.

A differenza di quanto era nelle possibilità di Emilio Sereni, oggi i paesaggi agrari, meglio i paesaggi rurali, possono essere utilizzati dal geografo come archivi della propria storia e nello stesso tempo come laboratori viventi, dove è possibile monitorare gli effetti ambientali delle pratiche attuali e interpretare le tracce delle pratiche pregresse che essi conservano ${ }^{36}$.

Emerge, grazie anche a queste nuove fonti, un vasto patrimonio di «paesaggi rurali di interesse storico» con inedite problematiche. Ad esempio paesaggi - spazi geografici concreti - dove si può riconoscere ancora attivo un più generale processo storico di «biodiversificazione ambientale ${ }^{37}$.

35. R. Cevasco, Memoria verde.

36. Sereni, come è noto, non è mai giunto, se non in margine nei suoi ultimi scritti, a formulare una archeologia del paesaggio agrario (M. Quaini, Paesaggi agrari; D. Moreno, O. Raggio, «Dalla storia del paesaggio agrario alla storia rurale»). Le formulazioni tarde, proposte dall'archeologia italiana, di una generica archeologia del paesaggio sono, dal punto di vista storico e geografico, assai discutibili e meriterebbero uno spazio a parte. Per i fondamenti di una archeologia rurale, che non può fare a meno di basi geografiche, si veda A. M. Stagno, "Archeologia rurale»; "Geografia degli insediamenti e risorse ambientali ".

37. R. Cevasco, D. Moreno, «Paesaggi rurali». 
Anche nelle applicazioni della geografia alla valorizzazione del patrimonio culturale ed ambientale si aprono nuove possibilità : in occasione del recente Catalogo Nazionale dei Paesaggi Rurali Storici promosso dal MIPAAF e coordinato a livello nazionale da Mauro Agnoletti, un gruppo di schede sono state dedicate a paesaggi specifici della montagna appenninica. In questa occasione - tappa di un importante processo di patrimonializzazione - attuali e precisi paesaggi rurali della montagna sono stati reinterpretati come eredità ambientali viventi di "paesaggi culturali» storici. Leggere oggi sul terreno questa eredità ambientale storica e i suoi meccanismi di perennizzazione è una proposta già scaturita, a scala europea, da uno dei molti progetti dedicati negli ultimi anni ai paesaggi rurali : il progetto ECL Our Common European Cultural Landscape Heritage promosso dall'Università di Bergen ${ }^{38}$.

L'esercizio necessario è quello - per riprendere la sintesi dei risultati fatta da Massimo Quaini - di decifrare le tracce materiali della storia del "paesaggio reale" come si sono conservate nella pluralità delle nuove fonti a disposizione del geografo, dello storico locale e dell'archeologo ambientale, rimettendo al centro del processo di patrimonializzazione il paesaggio geografico concreto anziché lo straripante "paesaggio mentale» e simbolico della geografia culturale ${ }^{39}$. Una geografia culturale che conosce una ininterrotta diffusione a livello europeo e che ha fortemente - quanto inconsapevolmente - orientato anche il progetto dell'Università di Bergen ${ }^{40}$. Per questo motivo, i casi studio cui si accenna in questa seconda parte ribadiscono la specificità di una lettura "ecologico-storica" delle fonti sostenuta dall'apporto dell'archeologia ambientale, avendo acquisito le nuove fonti osservazionali prodotte sul terreno (confrontando le procedure di osservazione geografica, naturalistica e archeologica) e sedimentarie ovvero che "anche nella vegetazione e nel suolo esiste una memoria da recuperare» ${ }^{41}$.

Tra i vari siti appenninici studiati presso il Laboratorio di Archeologia e Storia Ambientale in cui sono emerse relazioni rilevanti dal punto di vista

38. Framework Programme "Culture 2000», Action 2: Cultural Cooperation Agreements. Nel 2009 sono state pubblicate la versione inglese, tedesca e norvegese del volume che riassume i risultati del progetto PAN da cui è disceso il progetto ECL (K. Krzywinski, M. O’Connell, H. Küster (Hrsg.), Europäische Kulturlandschaften).

39. M. Quaini, "Poiché niente di quello che la storia sedimenta va perduto», p. 88.

40. R. Cevasco, D. Moreno, «Microanalisi geo-storica o geografia culturale della copertura vegetale?».

4I. M. Quaini, I paesaggi italiani, p. 153. 
dell'ecologia storica si possono ricordare i casi di studio dedicati ai sistemi di produzione multipla. È il caso dei popolamenti di ontano bianco nel sistema - così battezzato - dell'alnocoltura; il caso dei pascoli alberati di faggio e dei castagneti terrazzati e irrigui ${ }^{42}$. Un discorso a parte meriterebbero le zone umide, indagate come archivi biostratigrafici e come "perimetri d'acqua" per la ricostruzione della storia dei "paesaggi culturali» ${ }^{43}$ e i pascoli gestiti in funzione di produzioni casearie a scala familiare. Paesaggi che conservano comunque sequenze e stratificazioni storiche e memoria vivente - biologica ed ecologica - dei processi che li hanno attraversati anche quando, oggi, si trovano in condizioni post-colturali.

Rientra in questi paesaggi rurali in condizione post-colturale il caso di Montarlone, sito in un versante appenninico tra i 1200-1500 m slm nell'alta Val Trebbia genovese. Si tratta di una parcella relitta popolata da alcuni faggi capitozzati plurisecolari, eredità di un tipo di paesaggio culturale noto nella letteratura inglese come wooded meadow system, in cui l'arboratura presente viene ciclicamente e parzialmente privata della produzione fogliare a scopo foraggero (con la pratica del taglio a capitozza). A Montarlone - una volta individuato il funzionamento del sistema locale e delle sue pratiche - è stato possibile misurare le variazioni storiche di biodiversità del sito ${ }^{44}$ a partire da un diagramma pollinico ricavato da un profilo di suolo ${ }^{45}$. Si sono identificate nella sequenza dei pollini diverse fasi corrispondenti a diversi regimi agro-silvo-pastorali : dalla fase più recente che riflette l'abbandono del pascolo vaccino sino alla fase in cui era in funzione il pascolo alberato utilizzato prevalentemente da ovi-caprini. Nella stratigrafia dei pollini si ritrova la memoria della composizione in specie delle praterie che sono esistite in questo versante tra i grandi faggi sparsi del pascolo alberato a partire dall'alto medioevo. Tutte le fasi registrate

42. Solo per citare qualche esempio si vedano R. Cevasco, "The Environmental Heritage of a Past Cultural Landscape»; R. Cevasco, Ch. Molinari, «Microanalysis in Woodland Historical Ecology»; A. M. Stagno, "Geografia degli insediamenti e risorse ambientali»; R. Balzaretti, Ch. Watkins, M. Pearce (eds), Ligurian Landscapes.

43. Sul Progetto "Zone Umide» promosso dalla Direzione Regionale per i Beni Culturali e Paesaggistici della Liguria cfr. "Atti del Seminario Internazionale Wetlands as archives of the cultural landscapes: from research to management, Genoa, 29-30 January 2009», URL: http://www.dismec.unige.it/zum/atti.html, consultato il 10.02.2012.

44. R. Cevasco, D. Moreno, «Paesaggi rurali».

45. Ch. Molinari, Ricerche palinologiche per l'identificazione di sistemi agro-silvopastorali storici. 
nel diagramma pollinico hanno implicazioni con le dinamiche locali della biodiversità e, grazie alla documentazione testuale conservata negli archivi familiari, nell'archivio storico comunale e negli altri archivi che conservano materiale relativo a questo settore degli ex feudi imperiali appenninici, possono essere ricondotte ai diversi contesti sociali, economici e insediativi con una certa precisione per le fasi di età post-medievale. Ciò che si può sottolineare, sin da ora, è l'improvviso collasso (tab. 1) delle risorse erbacee del pascolo alberato (rappresentate nel diagramma dai pollini/popolamenti di Graminaceae, Plantago, Compositae ligulate, Umbelliferae), collasso particolarmente evidente nel diagramma a partire dal 1900 A.D. circa. Il fatto è direttamente correlabile, in questo spartiacque, con la caduta dei sistemi di transumanza ovina, un flusso di bestiame che è risalito stagionalmente su queste montagne dalla Riviera di Levante sino a memoria d'uomo. La fase post-colturale del sito di Montarlone, con l'abbandono generalizzato del pascolo bovino, dello sfalcio dei prati di altitudine, della ceduazione regolare dei boschi di faggio, perdura da quarant'anni ${ }^{46}$.

\begin{tabular}{|c|c|c|}
\hline c. $1960-70$ & sparizione pollini di: & $\begin{array}{l}\text { Graminaceae (il declino inizia con il 1800) } \\
\text { Plantago (ultimo picco intorno al 1930-40) } \\
\text { Compositae Liguliflorae (ultimo picco } \\
\text { intorno al 1930-40) } \\
\text { Trollius (ultimo picco intorno al 1950) } \\
\text { Umbelliferae }\end{array}$ \\
\hline c. $1930-40$ & $\begin{array}{l}\text { sparizione pollini di: } \\
\text { diminuzione pollini di: }\end{array}$ & $\begin{array}{l}\text { Caryophyllaceae } \\
\text { Compositae Tubuliflorae (ultimo picco } \\
\text { intorno al 1930-40) } \\
\text { Felci }\end{array}$ \\
\hline c. 1900 & sparizione pollini di: & Urticaceae \\
\hline c. 1800 & sparizione pollini di: & $\begin{array}{l}\text { Rumex } \\
\text { Biscutella }\end{array}$ \\
\hline
\end{tabular}

Tab. 1 - Tappe storiche di perdita della biodiversità (XVIII-XIX sec.) connesse al progressivo abbandono della gestione del pascolo alberato (R. Cevasco, D. Moreno, «Paesaggi rurali»). N.B. Le date sono ricostruite attraverso datazioni radioisotopiche.

46. Studi come questo di Montarlone, effettuati in diversi siti dello stesso spartiacque Trebbia-Aveto, sono stati recepiti nella pianificazione faunistico-venatoria della Provincia di Genova e hanno orientato progetti di valorizzazione e lo stesso piano di gestione del Sito di interesse comunitario «Roccabruna», in corso di elaborazione. 
Il secondo esempio si riferisce alla storia dell'adozione di una pianta foraggera nei sistemi colturali di una "villa» - nucleo di insediamento di montagna - dell'Appennino ligure orientale. Il paesaggio in oggetto è quello oggi dei prati-pascoli terrazzati e in parte alberati di cerri (Quercus cerris), interessati da un ciclo di seminativi e di riposo in funzione della produzione casearia domestica ${ }^{47}$ : la ricerca si è particolarmente riferita alle aziende familiari che in queste frazioni ancora oggi producono un formaggio con spiccate caratteristiche locali detto di S. Stefano d'Aveto.

Il nome geografico di questi prati-pascoli, come attribuito nel dialetto dai gruppi sociali che li utilizzano, è quello di «lupinelle» (dial.), derivato dal nome italiano (lupinella) adottato localmente per la leguminosa Onobrychis viciifolia ${ }^{48}$. La pianta è stata introdotta dalla Savoia dopo il 1820 nei "domestici» (dial.) (le «terre seminative» nella documentazione contemporanea) di questa valle: uno dei classici imprestiti dall'agronomia alta, ne è testimonianza, infatti, un appunto del viceintendente di Bobbio, il barone Alberto Nota (Torino 1775-1847). Dalle "terre seminative» la pianta entra progressivamente nei «selvatici» dell'alta Val d'Aveto ("terre alberate» di cerri) tra gli anni 1860 e 1920 , in corrispondenza con la fase di «miglioramento" dei pascoli per la produzione zootecnica intensiva che porterà lentamente a favorire la razza bovina "Bruna Alpina» a scapito della locale "Cabannina» e via via ad organizzare i primi caseifici sociali (a partire almeno dagli anni 1910). La ricerca, oltre a seguire il percorso della diffusione di questa pianta alla scala topografica (fino al suo ingresso nel dialetto) e a qualificare il contesto entro cui la sua coltivazione si inserisce, ha evidenziato le "esternalità positive» indotte dalle pratiche locali previste nel sistema colturale che tra gli anni 1990-2005 ha perennizzato l'ecologia di questi prati-pascoli (cioè ha consentito la riproduzione e conservazione) dei loro popolamenti vegetali e la presenza stessa della specie. Le azioni cicliche effettuate annualmente (rilevate negli anni 1997-2007) dai produttori

47. I paesaggi terrazzati dell'alta Val d'Aveto (GE) sono stati presentati all'attenzione dell'Unione Europea nel 2006 come "Patrimonio culturale europeo " attraverso il coinvolgimento della comunità di Alpicella (S. Stefano d'Aveto) reso possibile dalla fruttuosa collaborazione instaurata con i produttori locali nel corso delle ricerche decennali del LASA in questo settore della montagna appenninica (ECL Project, video Fields of Demeter, Università di Bergen, 2007).

48. Sulla storia di diffusione e regressione di questa foraggera in Europa occidentale tra XV e XIX secolo cfr. M. Ambrosoli, Scienziati, contadini e proprietari. 
sulla cotica erbacea (ripulitura invernale oggi con rastrello e non più con l'uso del fuoco controllato, sfalcio estivo, pascolo autunnale, ecc.) e sui cerri (scalvatura, una forma particolare di ceduazione per la produzione di foraggio fogliare) per il miglioramento della qualità del foraggio, pur nella loro discontinuità, hanno anche effetti indiretti (di attivazione) sulla composizione delle stazioni prative. Consentono la riproduzione di genziane, orchidee, funghi pregiati ("spinaroli», gen. Tricholoma), erbe commestibili; conservano habitat importanti per la piccola fauna stanziale come le lepri. Il risultato non è solo un miglioramento qualitativo della produzione foraggera ma anche l'attivazione di una serie di altre risorse ambientali, con un effetto complessivo misurabile di "esternalità positiva». Un risultato di estremo interesse nelle sue possibili applicazioni geografiche dato che i nuovi indirizzi della Politica Agraria Comunitaria sulla «condizionalità " considerano ormai indispensabile la presenza di simili effetti ambientali positivi per l'erogazione dei finanziamenti futuri alle aziende ${ }^{49}$.

Oggi, invece, le particelle in cui sono presenti popolamenti di Onobrychis, a causa della discontinuità delle pratiche locali di produzione, sono interessate da un progressivo processo di inselvatichimento che vede i prati "domestici» delle "lupinelle» trasformarsi in prati «selvatici» con peggioramento della qualità foraggera e perdita degli effetti di attivazione ${ }^{50}$.

L'«approccio storico microanalitico » ai problemi geografici consente dunque di analizzare il paesaggio rurale nella sua fisicità di spazio geografico concreto e nello stesso tempo di processo storico-ambientale. Il "Catalogo nazionale dei paesaggi rurali di interesse storico" risponde all'esigenza, urgente, di una gestione del patrimonio rurale in cui è considerata fondamentale la dimensione storica dei paesaggi e dunque il riferimento unificante è stato ancora agli studi pionieristici di Emilio Sereni ${ }^{51}$. Oggi le nuove fonti consentono al geografo di basare

49. Un altro punto di interesse riguarda il recupero di paesaggi agrari di interesse storico, comprensivi di pascoli e terrazzamenti, oggi coperti dal bosco a causa dell'abbandono. Un articolo del decreto legge sulla semplificazione recentemente approvato dal governo italiano stabilisce che tali paesaggi non sono più da considerarsi bosco e dunque il loro recupero è consentito senza incorrere in infrazioni alla legge forestale.

50. R. Cevasco, Memoria verde, p. 226-230.

5I. M. Quaini, Paesaggi agrari. 
la «significatività » storica dei paesaggi su una documentazione specifica attribuibile ad ogni paesaggio individuale.

I paesaggi rurali storici e la biodiversità che conservano, acquisita attraverso l'attivazione di habitat e risorse, una volta iscritti nel più vasto concetto di "patrimonio rurale vivente" e in una prospettiva europea (living rural heritage), rovesciano i problemi della loro gestione e conservazione quando espressi da una prospettiva puramente naturalistica. Un drammatico grafico costruito da Mauro Agnoletti sui dati raccolti per il Catalogo dimostra, infatti, che le aree protette e vincolate ai fini ambientali sono una delle maggiori cause della vulnerabilità dei paesaggi rurali di interesse storico quando si trovano localizzati al loro interno ${ }^{52}$.

Ancora una volta la montagna ligure emerge da queste ricerche applicate come un «laboratorio vivente» dove diventa sempre più pressante sperimentare il recupero di una gestione locale della biodiversità a partire dai saperi e saper-fare ancora presenti localmente. Resta da domandarsi se sia compito della ricerca geografica identificare e documentare la profondità che sta dietro a questi saperi e ai processi storici che hanno costruito i paesaggi rurali, la loro biodiversità e sostenibilità. Promuovere nuove politiche e nuove economie a partire dai micro-modelli che già funzionano pare, peraltro, una strada già imboccata ${ }^{53}$.

Roberta Cevasco

Università di Genova - Università del Piemonte Orientale

52. M. Agnoletti, Paesaggi Rurali Storici, p. 93, fig. 31-32.

53. Gli sforzi dovranno essere rivolti a contabilizzare il valore aggiunto dei sistemi produttivi locali in termini di "esternalità positive» indotte e a riattivare, nei contesti dove ancora resistono, quelle microistituzioni che storicamente si sono auto-organizzate localmente rivestendo un ruolo tecnico-politico nella gestione delle risorse collettive. Sul tema del community-based resource management si veda l'amplissima letteratura internazionale degli ultimi 20 anni sulla scia del lavoro di Elinor Ostrom (un esempio in URL: http://www.ecologyandsociety.org/vol15/iss4/art38/ consultato il 7.12.2012). 


\section{BIBLIOGRAFIA}

Agnoletti, Mauro (a cura di), Paesaggi Rurali Storici. Per un Catalogo Nazionale, Bari, Laterza, 2010.

Ambrosoli, Mauro, Scienziati, contadini e proprietari. Botanica e agricoltura nell'Europa occidentale 1350-1850, Torino, Einaudi, 1992.

Balée, William, Erickson, Clark, "Time, Complexity and Historical Ecology", in Time and Complexity in Historical Ecology. Studies in the Neotropical Lowlands, edited by William Balee and Clark Erickson, New York, Columbia University Press, 2006, p. 1-20.

Balzaretti, Ross, "Victorian Travellers, Apennine Landscapes and the Development of Cultural Heritage in Eastern Liguria, c. 1875-1914», History, 324/96 (2011), p. 436-458.

Balzaretti, Ross, Watkins, Charles, Pearce, Mark (eds), Ligurian Landscapes: Studies in Archaeology, Geography \& History, London, Accordia Research Institute/University of London, 2004.

Birks, Hilary H. et al. (eds), The Cultural Landscape. Past, Present and Future, Cambridge, Cambridge University Press, 1988.

Cevasco, Roberta, Memoria verde. Nuovi spazi per la geografia, Reggio Emilia, Edizioni Diabasis, 2007.

—, "The Environmental Heritage of a Past Cultural Landscape: The Alderwoods (Alnus incana Moench) in the Upper Aveto Valley (NW Apennines)", in Nature and History in Modern Italy, ed. by Marco Armiero, Marcus Hall, Athens, Ohio University Press, 2010, p. 126-140.

—, «Dopo Sereni: dal paesaggio agrario al patrimonio rurale. Le nuove fonti", in Paesaggi agrari. L'irrinunciabile eredità scientifica di Emilio Sereni, a cura di Massimo Quaini, Milano, Silvana Editoriale, 2011, p. 161-170.

Cevasco, Roberta, Molinari, Chiara, "Microanalysis in Woodland Historical Ecology. Evidences of Past Leaf Fodder Production in NW Apennines (Italy)", in Woodland Cultures in Time and Space: Tales from the Past, Messages for the Future, ed. by Eirini Saratsi et al., Athens, Embryo Publications, 2009, p. 147-154. 
Cevasco, Roberta, Moreno, Diego, «Microanalisi geo-storica o geografia culturale della copertura vegetale? Sull'eredità ambientale dei "paesaggi culturali" ", in Trame nello spazio 3. Quaderni di geografia storica e quantitativa, Firenze, All'Insegna del Giglio, 2007, p. 83-101.

—, "Paesaggi rurali: alle radici storiche della biodiversità», in Paesaggi Rurali Storici. Per un Catalogo Nazionale, a cura di Mauro Agnoletti, Bari, Laterza, 2010, p. 121-131.

Cevvasco, Roberta, Tigrino, Vittorio, "Lo spazio geografico concreto: una discussione tra storia politico-sociale ed ecologia storica", Quaderni Storici, 127 (2008), p. 207-242.

DA Pozzo, Carlo, "Bioetica e geografia», Rivista Geografica Italiana, 99/3 (1992), p. 503-513.

Dematteis, Giuseppe, "Nuovi percorsi della geografia umana in una storia non lineare", Quaderni Storici 127 (2008), p. 15-32.

Febvre, Lucien, La terre et l'évolution humaine. Introduction géographique à l'histoire, Paris, La Renaissance du Livre, 1922 (traduzione italiana Torino, Einaudi, 1980).

Grendi, Edoardo, Storia di una storia locale. L'esperienza ligure 17921992, Venezia, Marsilio, 1996.

Krzywinski, Knut, O’Connell, Michael, Küster, Hansjörg (Hrsg.), Europäische Kulturlandschaften. Wo Demeter ihre Felder hat und Pan zuhause ist, Bremen, Aschenbeck Media UG, 2009 (English and Norwegian editions, 2010).

Kunn, Thomas, The Structure of Scientific Revolutions, Chicago/London, The University of Chicago Press, 1962.

INGOLD, Alice, "Ecrire la nature. De l'histoire sociale à la question environnementale?», Annales HSS, 66/1 (2011), p. 11-29.

Marrone, Gianfranco, Addio alla Natura, Torino, Einaudi, 2011.

Molinari, Chiara, Ricerche palinologiche per l'identificazione di sistemi agro-silvo-pastorali storici, Tesi di Dottorato in Scienze storiche, indirizzo Geografia storica per la valorizzazione del patrimonio storico-ambientale, Genova, Università degli Studi di Genova, 2010.

Moreno, Diego, Dal documento al terreno. Storia e archeologia dei sistemi agro-silvo-pastorali, Bologna, Il Mulino-Ricerche, 1990.

Moreno, Diego, Montanari, Carlo, «Il lato "oscuro" del paesaggio: (per) una ecologia storica del paesaggio rurale italiano", in Riconquistare il paesaggio. La Convenzione Europea del Paesaggio 
e la Conservazione della Biodiversità in Italia, a cura di Corrado Teofili, Rosa Clarino, Roma, Stilgrafica, 2008, p. 159-175.

Moreno, Diego, Raggio, Osvaldo, «Dalla storia del paesaggio agrario alla storia rurale. L'irrinunciabile eredità scientifica di Emilio Sereni», Quaderni storici 100/1 (1999), p. 89-104.

Peterken, George Frederick, "Historical Approach to Woodland Ecology and Management", in Bedford Purlieus: Its History, Ecology and Management, edited by George Frederick Peterken, Robert Colin Welch, Huntingdon, Institute of Terrestrial Ecology, 1975, p. 3-4 (Monks Wood Experimental Station Symposium 7).

Quaini, Massimo, L'ombra del paesaggio. L'orizzonte di un'utopia conviviale, Reggio Emilia, Diabasis, 2006.

—, "Poiché niente di quello che la storia sedimenta va perduto", Quaderni Storici, 127 (2008), p. 55-108.

- (a cura di), "Una geografia per la storia. Dopo Lucio Gambi», Quaderni Storici, 127 (2008).

- (a cura di), I paesaggi italiani. Fra nostalgia e trasformazione, Rapporto annuale della Società Geografica Italiana, Genova, Brigati, 2009.

- (a cura di), Paesaggi agrari. L'irrinunciabile eredità scientifica di Emilio Sereni, Milano, Silvana Editoriale, 2011.

Raскнам, Oliver, Trees and Woodland in the British Landscape, London, Dent, 1976.

RagGio, Osvaldo, "Microhistorical Approaches to the History of Liguria: From Microanalysis to Local History. Edoardo Grendi's Achievements", in Ligurian Landscapes: Studies in Archaeology, Geography \& History, ed. by Ross Balzaretti, Charles Watkins, Mark Pearce, London, Accordia Research Institute/University of London, 2004, p. 97-103.

Russell, Emily W. B., People and the Land through Time. Linking Ecology and History, New Haven/London, Yale University Press, 1997.

Stagno, Anna Maria, "Archeologia rurale. Uno statuto debole», in Atti del $V$ Congresso nazionale di archeologia medievale, Foggia, 1-3 ottobre 2009, a cura di Pasquale Favia, Giuliano Volpe, Firenze, All'Insegna del Giglio, 2009, p. 20-25.

—, "Geografia degli insediamenti e risorse ambientali: un percorso tra fonti archeologiche e documentarie (Ventarola, Val d'Aveto, 
Rezzoaglio GE)", in Atti del convegno Geografie del Popolamento. Casi di studio, metodi e teorie. Grosseto 24-26 settembre 2008, a cura di Giancarlo Macchi Janica, 2009, p. 301-310.

Sturani, Maria Luisa, "Paesaggio e musei: la prospettiva della geografia", Rivista Geografica Italiana, 116/4 (2009), p. 379-402.

Torre, Angelo, «Un “tournant spatial” en histoire? Paysages, regards, ressources", Annales HSS, 63/5 (2008), p. 1127-1144.

-, Luoghi. La produzione di località in età moderna e contemporanea, Pomezia (Roma), Donzelli editore, 2011. 
